\title{
Simple largely tunable optical microcavity
}

A. Kiraz, A. Kurt, and M. A. DündarA. L. Demirel

Citation: Appl. Phys. Lett. 89, 081118 (2006); doi: 10.1063/1.2335371

View online: http://dx.doi.org/10.1063/1.2335371

View Table of Contents: http://aip.scitation.org/toc/apl/89/8

Published by the American Institute of Physics

\section{Articles you may be interested in}

Whispering-gallery mode microdisk lasers

Appl. Phys. Lett. 60, (1998); 10.1063/1.106688
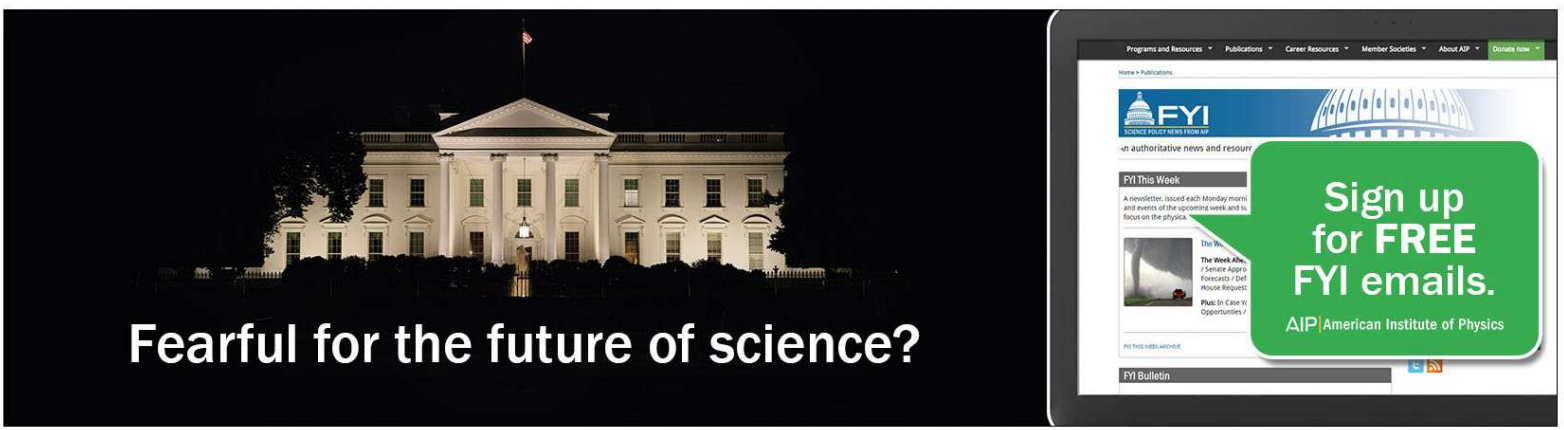


\title{
Simple largely tunable optical microcavity
}

\author{
A. Kiraz, ${ }^{\text {a) }}$ A. Kurt, ${ }^{\text {b) }}$ and M. A. Dündar \\ Department of Physics, Koç University, Rumelifeneri Yolu, Sariyer, Istanbul, 34450 Turkey \\ A. L. Demirel ${ }^{c)}$ \\ Department of Chemistry, Koç University, Rumelifeneri Yolu, Sariyer, Istanbul, 34450 Turkey
}

(Received 20 March 2006; accepted 30 June 2006; published online 24 August 2006)

\begin{abstract}
The authors demonstrate more than $9 \mathrm{~nm}$ tunability of the whispering gallery modes (WGMs) of rhodamine B doped water microdroplets resting on a superhydrophobic surface. Tunability was achieved by controlling the size of the microdroplets in a current controlled mini humidity chamber. WGMs were observed with quality factors of more than 8000 when kept stable. The sensitivity of the resonances to the size and shape of the microdroplet reveals opportunities for the use of this technique as a probe to characterize superhydrophobic surfaces and investigate liquid-solid surfaces. (C) 2006 American Institute of Physics. [DOI: 10.1063/1.2335371]
\end{abstract}

Optical microcavities find various applications in both fundamental and applied research areas such as cavity quantum electrodynamics, optoelectronics, and biological sensing. ${ }^{1}$ They possess high quality and low volume resonances, which yield large coupling constants to a resonant dipole emitter. This has led to notable demonstrations in quantum optics among which are very low threshold lasers, ${ }^{2}$ Purcell effect, ${ }^{3}$ and vacuum Rabi splitting. ${ }^{4}$ Maximum $0.7 \mathrm{~nm}$ tunability was achieved by changing temperature in experiments performed at cryogenic temperatures. ${ }^{5-7}$

Applications of optical microcavities in optical communication systems based on wavelength division multiplexing have been demonstrated by various studies using semiconductor or polymer microdisk and microring microcavities. ${ }^{8}$ Despite the inherent potential, microcavity based devices have not found wide use in modern optoelectronic networks. One of the main obstacles has been tunability. Refractive index control of the constituent materials in a solid microcavity by changing the temperature ${ }^{9,10}$ or the electric field applied to liquid crystal cladding layers ${ }^{11}$ enabled limited tunability within a maximum spectral range of $0.25 \mathrm{~nm}$.

Controlling size and shape an alternative mechanism for tuning microcavity resonances. Using solid optical microcavities a maximum tunability of $\sim 1 \mathrm{~nm}$ was demonstrated under a large external force. ${ }^{12}$ In contrast, a liquid microcavity provides relatively easy control of its shape due to its viscous nature. An applied external force, electric field, or control of the evaporation/condensation kinetics can induce size and shape changes in liquids. Liquid lenses, in which electrical field is used to control the shape, have even found technological applications. ${ }^{13}$

The tendency of liquid droplets to minimize interfacial energy by minimizing the interfacial area results in nearly spherical shapes in air. This inspired their use as microsphere microcavities. In their pioneering work, Ashkin and Dziedzic used suspending single liquid microdroplets in air by optical levitation. ${ }^{14}$ They observed whispering gallery modes (WGMs) by analyzing the laser power required to stabilize the height of the microdroplets as a function of wavelength. ${ }^{15}$

\footnotetext{
${ }^{\text {a)} E l e c t r o n i c ~ m a i l: ~ a k i r a z @ k u . e d u . t r ~}$

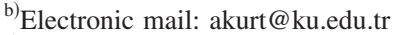

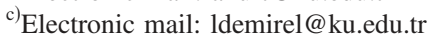

In these experiments, evaporation also led to the spectral drift of the WGMs. Liquid droplet characterization for diagnostic purposes was also developed for a wide range of sizes, and selected imaging of various liquid phase constituents of a spray using two-dimensional fluorescence, lasing, and stimulated Raman scattering was reported. ${ }^{16}$

In this letter we demonstrate the potential of a nearly spherical water microdroplet on a superhydrophobic surface as a largely tunable optical microcavity. Evaporation/ condensation kinetics were employed to tune the WGMs of these microcavities. In contrast to the microdroplets suspended in air, no additional technique such as optical levitation is required for position stabilization.

Superhydrophobic surfaces have high water repellency due to the combined effect of hydrophobicity and surface roughness. ${ }^{17}$ The contact angle of a millimeter sized water droplet on such a surface is larger than $150^{\circ}$ (contact angle being $180^{\circ}$ for a perfect sphere). Superhydrophobic surfaces in our study were prepared by spin coating silica nanoparticles having hydrophobic surface coatings (Degussa AG, Aeroxide LE1) on glass substrates. ${ }^{18}$ The resulting surfaces had nanometer scale surface roughness and were transparent to visible light. The average contact angle of water droplets of $\sim 2 \mathrm{~mm}$ diameter on the superhydrophobic surface was measured to be $152.6^{\circ}$. For $10-20 \mu \mathrm{m}$ diameter water microdroplets, the average contact angle is expected to be $1-2^{\circ}$ smaller than that of milimeter sized droplets due to Gibbs contact line tension. ${ }^{19}$ In our samples, the microdroplets exhibited small contact angle hysteresis and moved easily on the surface when disturbed, an indication of being in the Cassie-Baxter regime of superhydrophobicity. ${ }^{20}$ The effects of droplet size and surface roughness, especially for micron sized droplets and nanometer scale roughness, on the motion of liquid droplets on solid surfaces are important in microfluidic applications, ${ }^{21}$ and are yet to be understood fundamentally. ${ }^{19,22}$ Being sensitive to nanometer scale size and shape changes, WGMs of a microdroplet on a solid surface also serve as a probe for the investigation of the dynamics at fluid/solid interfaces.

A home-built current controlled mini humidity chamber was used throughout the experiments [Fig. 1(a)]. The chamber stood on the superhydrophobic surface, and an attached nebulizer generates microdroplets doped with $20 \mu \mathrm{M}$ 


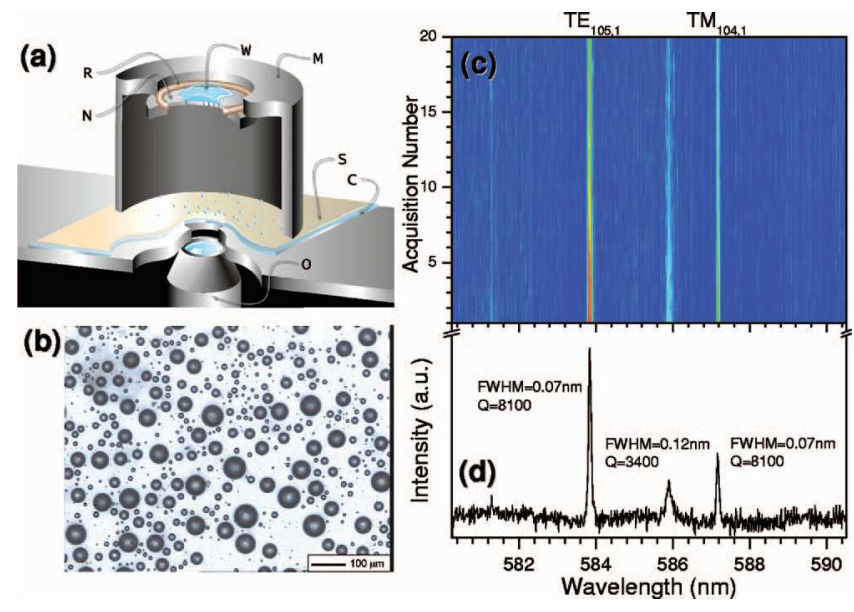

FIG. 1. (Color) (a) Illustration of the experimental setup. N, ultrasonic nebulizer; R, nichrome resistor; $\mathrm{W}$, reservoir of rhodamine $\mathrm{B}$ doped water; $\mathrm{M}$, mini chamber; $\mathrm{S}$, superhydrophobic surface; $\mathrm{C}$, cover glass; $\mathrm{O}$, microscope objective. (b) Top view of water microdroplets on the superhydrophobic surface. (c) Contour plot of the emission spectra taken from a microdroplet with a radius of $6 \mu \mathrm{m}$ showing stable WGMs. Intensity values in arbitrary units increase from blue to red. (d) Sum of the 20 acquisitions in (c). The peaks at 583.85 and $587.17 \mathrm{~nm}$ are identified as $\mathrm{TE}_{105,1}$ and $\mathrm{TM}_{104,1}$ WGMs, respectively.

rhodamine $\mathrm{B}$ ranging from a few up to $\sim 30 \mu \mathrm{m}$ in diameter [Fig. 1(b)]. WGMs were observed by the fluorescence spectrum collected from single microdroplets. ${ }^{23}$ High quality and low volume WGMs enhance the rhodamine B emission at resonant wavelengths, hence enabling their observation. Joule heating of a $3 \Omega$ nichrome wire resistor was used to control the ambient humidity level of the chamber, causing evaporation or condensation in microdroplets. Heating currents between 0.15 and $0.3 \mathrm{~A}$ were used to obtain stable or nearly stable microdroplets. Although the stabilizing heating current was not constant between the experiments, fine tuning with 0.01 A sensitivity helped to keep the size of the microdroplets highly stable. Additional parameter for local stability control was laser power absorbed by microdroplets. ${ }^{24}$

A continuous wave solid-state laser $(\lambda=532 \mathrm{~nm})$ was used to excite the microdroplets within resolution limited spots near the outer surface. Excitation of microdroplets and collection of the fluorescence were done through a high numerical aperture microscope objective $(60 \times, \mathrm{NA}=1.4)$ in the inverted geometry. After being transmitted through a dichroic mirror (Q570LP), and a $1.5 \times$ magnifier element, the collected fluorescence was dispersed using a $50 \mathrm{~cm}$ monochromator (spectral resolution of $\sim 0.06 \mathrm{~nm}$ ) and detected by a charge coupled device camera. All the emission spectra presented in this letter were taken using an exposure time of $2 \mathrm{~s}$, under excitation intensities between 2 and $5 \mu \mathrm{W}$. Each exposure of the camera was followed by $2.14 \mathrm{~s}$ readout time.

Figures 1(c) and 1(d) show high resolution spectra obtained from a sizewise stable microdroplet. The temporal sta- bility of the microcavity is demonstrated in the contour plot [Fig. 1(c)] and in the integrated spectrum [Fig. 1(d)]. During $82.8 \mathrm{~s}$ of data acquisition no spectral drift was observed in the WGMs within the resolution of the spectrometer setup $(0.06 \mathrm{~nm})$. This corresponds to a size stability of $\sim 1 \mathrm{~nm}$. The decrease in the intensity of the WGMs between consecutive acquisitions was due to photobleaching of rhodamine B molecules. Under the assumption of a dielectric sphere in air, the observed WGMs are identified by two linearly independent solutions to the Helmholtz equation with a mode number $n$ and mode order $r$ (TE ${ }_{n, r}$ or $\left.\mathrm{TM}_{n, r} \mathrm{WGMs}\right){ }^{23}$ For the spectra shown in Fig. 1, we identified the WGMs located at 583.85 and $587.17 \mathrm{~nm}$ as $\mathrm{TE}_{105,1}$ and $\mathrm{TM}_{104,1}$, respectively. The peak at $585.90 \mathrm{~nm}$ with a relatively large full width at half maximum (FWHM) should stem from a WGM with a high mode order. In the low pumping regime where stimulated emission is negligible as compared to spontaneous emission, the quality factors ( $Q$ factors) of the WGMs are given by the FWHMs of the observed peaks. This condition was satisfied in our experiments with the selected excitation intensity $(2-5 \mu \mathrm{W})$, which was much smaller than the saturation power of the $20 \mu \mathrm{M}$ rhodamine B solution. In Fig. 1(d), a $Q$ factor of 3400 was observed for the peak at $585.90 \mathrm{~nm}$, while the $Q$ factors of the $\mathrm{TE}_{105,1}$ and $\mathrm{TM}_{104,1}$ WGMs were determined to be larger than 8000, which is almost the resolution limit of our experimental setup. Considering the microdroplet radius of $7 \mu \mathrm{m}$, the free spectral range (FSR) of the WGMs is expected to be larger than $7 \mathrm{~nm},{ }^{25}$ yielding a minimum finesse (FSR/FWHM) of $\sim 100$ for the high quality WGMs.

Proof of negligible stimulated emission is also evident in the acquired high resolution spectra. Relative intensities of the three WGMs in Figs. 1(c) and 1(d) were preserved during the acquisitions despite significant photobleaching. Hence no lasing threshold was observed between the correponding acquisitions. The fact that the low quality WGM had an intensity comparable to the intensities of the two high quality WGMs is another evidence for the absence of lasing. According to Ref. 16 the lasing modes should be redshifted, which we did not observe.

WGMs were tuned in the directions of both evaporation and condensation as a function of the ambient humidity. Figure 2 shows the consecutive spectra taken from three different microdroplets. Evaporation was observed in Fig. 2(a) leading to a total spectral drift of $3.1 \mathrm{~nm}$, whereas in Fig. 2(b) condensation caused a total spectral drift of $9.6 \mathrm{~nm}$ in the WGMs. In Fig. 2(c), the direction of evaporation was reversed by the sudden increase of local humidity as a result of an increase in the heating current from 0.28 to $0.31 \mathrm{~A}$. After a spectral drift by $2.6 \mathrm{~nm}$ in the direction of evaporation, a spectral drift of $3.3 \mathrm{~nm}$ was observed in the direction of condensation.

For an ideal sphere with a large structural factor $(x=2 \pi a / \lambda)$, FSR is inversely proportional to the radius, $a .^{25}$

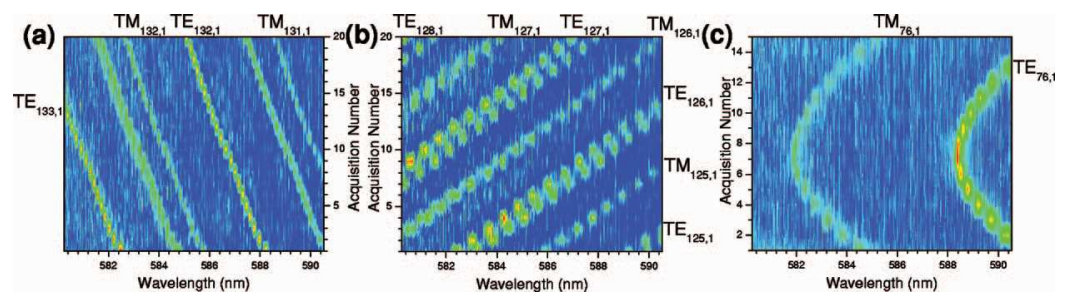

FIG. 2. (Color) Consecutive spectra taken from three microdroplets exhibiting (a) evaporation, (b) condensation, and (c) evaporation followed by condensation. The radii of the microdroplets were 9,8 , and $5 \mu \mathrm{m}$ in (a), (b), and (c), respectively. Identified WGMs are shown on the contour plots. The intensity values in arbitrary units increase from blue to red. 


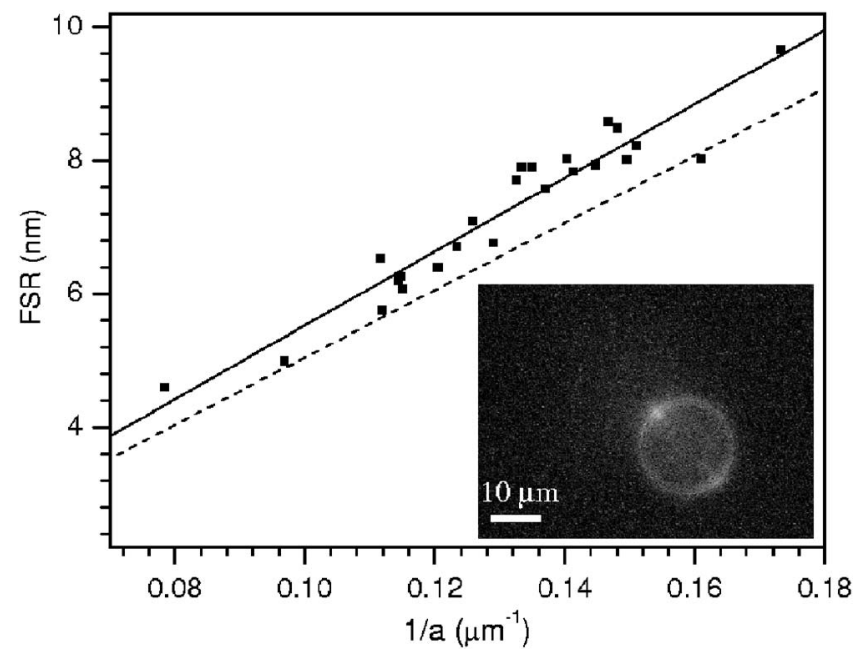

FIG. 3. (a) FSR of WGMs as a function of the reciprocal radii of the microdroplets. The dashed line is the expected dependence for ideal water microspheres in air. The solid line is the best fit to the experimental data. The linear dependence reflects nearly spherical geometry of the water microdroplets. (b) Fluorescence image of a water microdroplet. Characteristic ring shape of the WGMs is visible.

Figure 3 depicts the dependence of the observed FSRs as a function of the reciprocal radii of microdroplets. For each measured FSR, the radius of the microdroplet was determined from fluorescence optical images as seen in the inset in Fig. 3. The revealed linearity between FSR and $1 / a$ is an indication of the nearly spherical geometry of the microdroplets. The difference between the best fit to experimental data (solid line) and the expected radius dependence for ideal water microspheres in air (dashed line) reflects ${ }^{25}$ the deviation from an ideal sphere to a truncated spherical shape ${ }^{19}$ on the superhydrophobic surface.

In this letter a water microdroplet resting on a superhydrophobic surface was introduced as a largely tunable optical microcavity. For future applications, faster tuning methods can be developed with an applied external force or electric field. ${ }^{26}$ Enhancement of optical field intensities in the water microcavity might lead to applications in fluid sensing or precise measurements of optical interactions in minute samples of biological specimens or biomolecules. The measurement technique presented here can also serve as a useful probe in characterizing the dynamics at liquid/solid interfaces due to its high sensitivity to the size and shape of microdroplets.

This work was supported by the Alexander von Humboldt Foundation and the Scientific and Technological
Research Council of Turkey (Grant No. TÜBİTAK105T500). The authors thank F. Menzel for providing the silica nanoparticles and S. Saçıldı for graphical illustration. Two of the authors (A. L. Demirel and A. Kiraz) acknowledge the financial support of the Turkish Academy of Sciences in the framework of the Young Scientist Award program (Grant Nos. EA/TÜBA-GEBIP/2001-1-1 and A.K/ TÜBA-GEBIP/2006-19).

${ }^{1}$ K. J. Vahala, Nature (London) 424, 839 (2003).

${ }^{2}$ V. Sandoghdar, F. Treussart, J. Hare, V. Lefevre-Seguin, J.-M. Raimond, and S. Haroche, Phys. Rev. A 54, R1777 (1996).

${ }^{3}$ J. M. Gérard, B. Sermage, B. Gayral, B. Legrand, E. Costard, and V. Thierry-Mieg, Phys. Rev. Lett. 81, 1110 (1998).

${ }^{4}$ G. Khitrova, H. M. Gibbs, M. Kira, S. W. Koch, and A. Scherer, Nat. Phys. 2, 81 (2006).

${ }^{5}$ J. P. Reithmaier, G. Sek, A. Löffler, C. Hofmann, S. Kuhn, S. Reitzenstein, L. V. Keldysh, V. D. Kulakovskii, T. L. Reinecke, and A. Forchel, Nature (London) 432, 197 (2004).

${ }^{6}$ T. Yoshie, A. Scherer, J. Hendrickson, G. Khitrova, H. M. Gibbs, G. Rupper, C. Ell, O. B. Shchekin, and D. G. Deppe, Nature (London) 432, 200 (2004).

${ }^{7}$ A. Kiraz, P. Michler, C. Becher, B. Gayral, A. İmamoğlu, L. Zhang, E. Hu, W. V. Schoenfeld, and P. M. Petroff, Appl. Phys. Lett. 78, 3932 (2001).

${ }^{8}$ S. C. Hagness, D. Rafizadeh, S. T. Ho, and A. Taflove, J. Lightwave Technol. 15, 2154 (1997).

${ }^{9}$ P. Heimala, P. Katila, J. Aarnio, and A. Heinämäki, J. Lightwave Technol. 14, 2260 (1996)

${ }^{10}$ D. Armani, B. Min, A. Martin, and K. J. Vahala, Appl. Phys. Lett. 85, 5439 (2004)

${ }^{11}$ B. Maune, R. Lawson, C. Gunn, A. Scherer, and L. Dalton, Appl. Phys. Lett. 83, 4689 (2003).

${ }^{12}$ W. von Klitzing, R. Long, V. S. Ilchenko, J. Hare, and V. Lefevre-Seguin, New J. Phys. 3, 14 (2001).

${ }^{13}$ S. Kuiper and B. H. W. Hendricks, Appl. Phys. Lett. 85, 1128 (2004).

${ }^{14}$ A. Ashkin and J. M. Dziedzic, Science 187, 1073 (1975).

${ }^{15}$ A. Ashkin and J. M. Dziedzic, Phys. Rev. Lett. 38, 1351 (1977).

${ }^{16}$ A. Serpengüzel, J. C. Swindal, R. K. Chang, and W. P. Acker, Appl. Opt. 31, 3543 (1992).

${ }^{17}$ A. Lafuma and D. Quere, Nat. Mater. 2, 457 (2003).

${ }^{18}$ M. Y. Yüce, A. L. Demirel, and F. Menzel, Langmuir 21, 5073 (2005).

${ }^{19}$ A. Checco, P. Guenoun, and J. Daillant, Phys. Rev. Lett. 91, 186101 (2003).

${ }^{20}$ G. McHale, N. J. Shirtcliffe, and M. I. Newton, Langmuir 20, 10146 (2004).

${ }^{21}$ J. Atencia and D. J. Beebe, Nature (London) 437, 648 (2005).

${ }^{22}$ S. M. M. Ramos, E. Charlaix, A. Benyagoub, and M. Toulemonde, Phys. Rev. E 67, 031604 (2003).

${ }^{23}$ R. E. Benner, P. W. Barber, J. F. Owen, and R. K. Chang, Phys. Rev. Lett. 44, 475 (1980).

${ }^{24}$ J. D. Eversole, H.-B. Lin, and A. J. Campillo, J. Opt. Soc. Am. B 12, 287 (1995).

${ }^{25}$ S.-X. Qian, J. B. Snow, H. M. Tzeng, and R. K. Chang, Science 231, 486 (1986).

${ }^{26}$ V. H. Kwong, M. A. Mossman, and L. A. Whitehead, Appl. Opt. 43, 808 (2004). 